\title{
Validation of the Reduced Vector Potential Formulation with the DtN Boundary Condition
}

\author{
Anton EFREMOV ${ }^{\mathrm{a}, 1}$, Lalita UDPA ${ }^{\mathrm{a}}$ and Antonello TAMBURRINO ${ }^{\mathrm{b}}$ \\ ${ }^{a}$ Michigan State University, East Lansing, MI 48824, USA \\ ${ }^{\mathrm{b}}$ University of Cassino and Southern Lazio, Cassino, Italy
}

\begin{abstract}
This work describes the validation against experimental data of a Reduced Vector Potential Formulation combined with a boundary condition given by the Dirichlet-to-Neumann operator. One of the classic nondestructive testing problems is investigated: the differential bobbin coil scan inside a tube with a defect. Several defects are simulated, and the results are compared to the experimental data acquired at four frequencies.
\end{abstract}

Keywords. Boundary Conditions, Eddy Currents, Finite Element Analysis, Nondestructive Testing

\section{Introduction}

Eddy current systems play an important role in the modern nondestructive testing. An ability to provide an accurate simulation of an eddy current inspection procedure is crucial to multiple applications, including sensor design and optimization, defect characterization, and many others.

A prominent way to set up an eddy current simulation is to utilize one of the differential formulations for the low-frequency approximation of Maxwell's equations and then solve it within a finite element framework ([1], [2]). In this case, the computation domain needs to be truncated and an appropriate boundary condition needs to be set. One way of imposing a boundary condition is to apply a Dirichlet-to-Neumann operator on the truncation boundary, as in [3], [4], [5], [6]. This boundary condition is exact, which means that it does not introduce any error in the physics on the truncation boundary and thus allows to put it close to the investigated object.

The authors previously derived a Reduced Vector Potential formulation with a DtN boundary condition in [7], [8], [9]. This paper contains a brief summary of the proposed formulation and its numerical validation against an experimental data for a typical ECT problem: a coil scan inside a conducting tube that has a volumetric defect in the wall.

\footnotetext{
${ }^{1}$ Corresponding Author: Anton Efremov, Nondestructive Evaluation Laboratory, Michigan State University, College of Engineering, 428 S. Shaw Lane, Room 2580, East Lansing, MI 48824-1226, USA; E-mail: efremova@egr.msu.edu.
} 


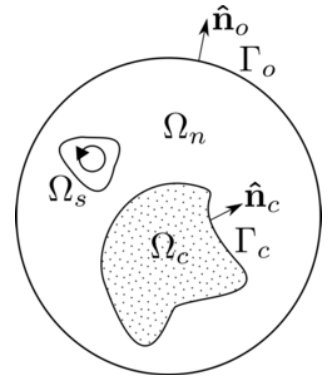

(a)

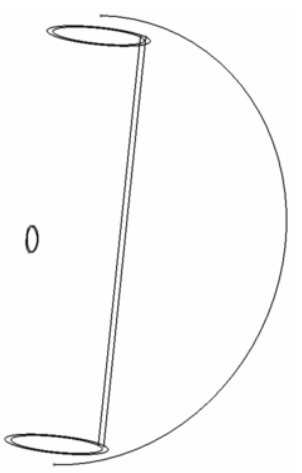

(b)

Figure 1. (a) The schematic diagram of the domain $\Omega$, (b) The sketch of the simulated problem geometry: conducting tube with a defect

\section{Formulation}

\subsection{Governing equations}

The computational region is defined as $\Omega=\Omega_{n} \cup \Omega_{c}$, where $\Omega_{n}$ and $\Omega_{c}$ are the nonconducting and conducting regions respectively (see Figure 1a). The truncation boundary of $\Omega$ is denoted as $\Gamma_{o}=\partial \Omega$, and $\hat{\boldsymbol{n}}_{o}$ is an outward-looking normal to $\Gamma_{o}$.

In low frequency approximation the displacement current is neglected, and Maxwell's equations, that govern the underlying physics, take the following form ("magnetoquasistatic limit"):

$$
\begin{array}{rlrl}
\nabla \times \boldsymbol{E} & =-j \omega \boldsymbol{B} & \text { in } \Omega \\
\nabla \cdot \boldsymbol{B} & =0 & \text { in } \Omega \\
\nabla \times \boldsymbol{H} & =\boldsymbol{J} & \text { in } \Omega \\
\boldsymbol{H} & =v \boldsymbol{B} & \text { in } \Omega \\
\boldsymbol{J} & =\sigma \boldsymbol{E} & & \text { in } \Omega_{c}
\end{array}
$$

Here symbols $\boldsymbol{D}, \boldsymbol{B}, \boldsymbol{E}, \boldsymbol{H}, \boldsymbol{J}$ denote electric and magnetic flux, electric field, magnetic field and current density respectively. Symbols $\mu, v, \sigma$ represent magnetic permeability, magnetic reluctivity and conductivity, $\omega$ is an angular frequency and $j^{2}=-1$. The magnetic vector potential $\boldsymbol{A}$ and (modified) electric scalar potential $\phi$ are defined as follows:

$$
\begin{aligned}
& \boldsymbol{B}=\nabla \times \boldsymbol{A} \\
& \boldsymbol{E}=-j \omega \boldsymbol{A}-j \omega \nabla \boldsymbol{\phi}
\end{aligned}
$$

The source current $\boldsymbol{J}_{s}$ resides in a source region $\Omega_{s} \subset \Omega_{n}$ and generates the source magnetic field $\boldsymbol{H}_{s}$ and vector potential $\boldsymbol{A}_{s}$. Now the magnetic vector potential could be represented as $\boldsymbol{A}=\boldsymbol{A}_{r}+\boldsymbol{A}_{s}$, where $\boldsymbol{A}_{r}$ is the reduced vector potential, generated by eddy 
currents in the conductor. This decomposition could be utilized to obtain the following system of equations from Eqs.(1)-(5) - the reduced vector potential formulation in weak form ([1], [2]):

$$
\begin{array}{r}
\int_{\Omega}\left(\nabla \times \boldsymbol{N}_{n}\right) \cdot\left(v \nabla \times \boldsymbol{A}_{r}\right) d \Omega+j \omega \int_{\Omega} \boldsymbol{N}_{n} \cdot \sigma \boldsymbol{A}_{r} d \Omega+j \omega \int_{\Omega} \boldsymbol{N}_{n} \cdot \sigma \nabla \phi d \Omega+ \\
+\int_{\Gamma_{o}} \boldsymbol{N}_{n} \cdot\left(\hat{n}_{o} \times v \nabla \times \boldsymbol{A}_{r}\right) d S=-\int_{\Omega}\left(\nabla \times \boldsymbol{N}_{n}\right) \cdot\left(v_{r}-1\right) \boldsymbol{H}_{s} d \Omega-j \omega \int_{\Omega} \boldsymbol{N}_{n} \cdot \sigma \boldsymbol{A}_{s} d \Omega \\
-j \omega \int_{\Omega} \nabla \phi_{n} \cdot \sigma \boldsymbol{A}_{r} d \Omega-j \omega \int_{\Omega} \nabla \phi_{n} \cdot \sigma \nabla \phi d \Omega=j \omega \int_{\Omega} \nabla \phi_{n} \cdot \sigma \boldsymbol{A}_{s} d \Omega
\end{array}
$$

Here $N_{k}$ and $\phi_{k}$ are the test functions. With the use of basis functions $N_{k}{ }^{\prime} \in$ $H(\operatorname{curl}, \Omega)$ and $\phi_{k}{ }^{\prime} \in H(\operatorname{grad}, \Omega)$, we can approximate $\boldsymbol{A}_{r}=\sum_{k=1}^{N_{A}} \alpha_{k} N_{k}{ }^{\prime}$ and $\phi=\sum_{k=1}^{N_{\phi}} \beta_{k} \phi_{k}{ }^{\prime}$, where $N_{A}$ and $N_{\phi}$ stand for the number of basis functions, $\alpha_{k}$ and $\beta_{k}$ are unknown coefficients. This expansion is then substituted into the Eqs.(8)-(9), and when we set $N_{k}=N_{k}{ }^{\prime}$ and $\phi_{k}=\phi_{k}{ }^{\prime}$, the square system of linear equations is obtained. An appropriate boundary condition on $\Gamma_{o}$ will make this system complete.

\subsection{Dirichlet-to-Neumann operator on the sphere}

In our case, the Dirichlet-to-Neumann operator $\Lambda$ could be defined as an operator that acts on the solution of the vector Laplace equation in source free space and maps the tangential part of the reduced vector potential $\boldsymbol{A}_{r}$ on $\Gamma_{o}$ to the tangential part of its curl:

$$
\Lambda:\left(\boldsymbol{A}_{r}\right)_{t} \rightarrow\left(\nabla \times \boldsymbol{A}_{r}\right)_{t} \text { on } \Gamma_{o}
$$

Within FEM framework, an operator $\Lambda$ could be utilized to build a boundary condition in a following way. First, we decompose the unknown vector potential on $\Gamma_{o}$ into FEM basis $N_{k}$ and $\phi_{k}$. Then, this decomposition is converted to a tangential curl $\left(\nabla \times \boldsymbol{A}_{r}\right)_{t}$ with a use of $\Lambda$. The obtained expansion is then substituted into Eq.(8).

If the computatinal domain boundary $\Gamma_{o}$ is a sphere, analytic form of the operator $\Lambda$ is available ([7], [8], [9]). If the radius of the sphere is $R$ and $\boldsymbol{U}_{l m}, \boldsymbol{V}_{l m}$ denote the tangential vector spherical harmonics (see [10]), the following expansion could be derived:

$$
\int_{\Gamma_{o}} \boldsymbol{N}_{n} \cdot\left(\hat{n}_{o} \times v \nabla \times \boldsymbol{A}_{\boldsymbol{r}}\right) d S=\sum_{k=1}^{N_{A}} \alpha_{k} \sum_{l m} \frac{l R}{\mu_{0}}\left[\frac{\left\langle\boldsymbol{N}_{k}, \boldsymbol{U}_{l m}\right\rangle\left\langle\boldsymbol{N}_{n}, \boldsymbol{U}_{l m}\right\rangle}{\left\|\boldsymbol{U}_{l m}\right\|^{2}}-\frac{\left\langle\boldsymbol{N}_{k}, \boldsymbol{V}_{l m}\right\rangle\left\langle\boldsymbol{N}_{n}, \boldsymbol{V}_{l m}\right\rangle}{\left(R^{2} \| \boldsymbol{V}_{l m}||\right)^{2}}\right]
$$

The addition of this term completes the system linear equations obtained from Eqs.(8)-(9). 


\section{Numerical results}

\subsection{Simulation setup}

The investigated problem is a coil scan inside the conductive tube with a volumetric defect inside the wall. The tube is oriented along the $z$ axis. The 3D test geometry of the problem is shown in Figure $1 \mathrm{~b}$ and the $x$ - $z$ cross-section of the tube is displayed in Figure 2 . The tube has an outer diameter of $19.0 \mathrm{~mm}$, the wall thickness of $1.1 \mathrm{~mm}$ and is made of non-magnetic material with $\mu_{r}=1$ characterized by its conductivity of $\sigma=8.71 \times 10^{5}$ $\mathrm{S} / \mathrm{m}$ (equivalent resistivity value $\rho=114.8 \mu \Omega$-cm).

Four volumetric defects were simulated: the flat bottom holes $(\mathrm{FBH})$ with diameters of $4.8 \mathrm{~mm}, 4.8 \mathrm{~mm}, 2.8 \mathrm{~mm}, 1.3 \mathrm{~mm}$ and depths of $0.22 \mathrm{~mm}$ ( $20 \%$ tube wall, TW), $0.44 \mathrm{~mm}$ $(40 \% \mathrm{TW}), 0.66 \mathrm{~mm}(60 \% \mathrm{TW})$ and a through-wall hole, correspondingly. The defects were positioned on the outer side of the tube.

The source field was generated by two geometrically identical coils that were carrying the total current of $1 \mathrm{~A}$ each and a phase difference of $180^{\circ}$. This represents the differential measurement [11]: in the section of the tube away from the defect the induced voltages in the coils have equal magnitude (so that the resulting impedance of the two coil system is zero), whereas in proximity of the defect the induced voltages in the two coils are different, which results in a non-zero signal that could be used to detect the presence of the defect. The simulated coil parameters were the following: outer diameter of $15.2 \mathrm{~mm}$ and cross-section of $1.5 \mathrm{~mm}$ by $1.3 \mathrm{~mm}$. The spacing between the coils is $1.5 \mathrm{~mm}$. The coil lift-off in this case equals to $0.8 \mathrm{~mm}$. The simulation of the model result in a one-dimensional array of complex voltages evaluated in every scan position.

The length of the simulated tube segment was $76 \mathrm{~mm}$. The computational domain was a sphere of radius $40 \mathrm{~mm}$. It was discretized into unstructured non-uniform tetrahedral mesh generated by Gmsh software [12]. Mesh density was set to be approximately 12 elements per wall in the defect area and 6 elements per wall thickness everywhere else.

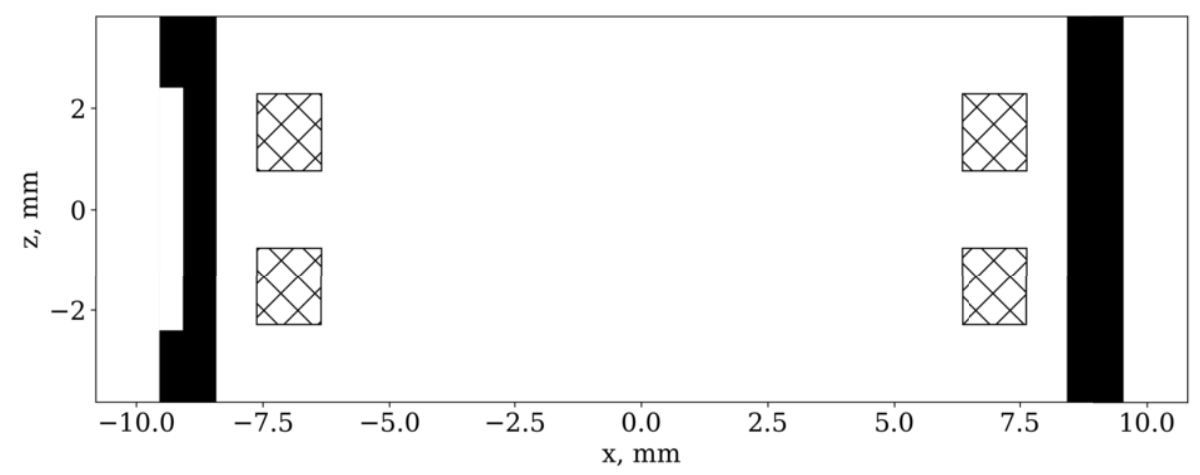

Figure 2. Geometry of the problem: $40 \% \mathrm{TW}$ defect 


\begin{tabular}{|llcc|}
\hline Frequency, kHz & Defect Type & Phase difference, deg & Peak voltage error, \% \\
\hline 35 & $20 \% \mathrm{FBH}$ & 4 & 11.00 \\
35 & $40 \% \mathrm{FBH}$ & 2 & 10.00 \\
35 & $60 \% \mathrm{FBH}$ & -1 & 3.00 \\
35 & $100 \% \mathrm{FBH}$ & 0 & 0.00 \\
140 & $20 \% \mathrm{FBH}$ & -4 & 8.00 \\
140 & $40 \% \mathrm{FBH}$ & -3 & 7.00 \\
140 & $60 \% \mathrm{FBH}$ & 5 & 14.00 \\
140 & $100 \% \mathrm{FBH}$ & 0 & 0.00 \\
280 & $20 \% \mathrm{FBH}$ & -16 & 14.00 \\
280 & $40 \% \mathrm{FBH}$ & -9 & 3.00 \\
280 & $60 \% \mathrm{FBH}$ & -6 & 3.00 \\
280 & $100 \% \mathrm{FBH}$ & 0 & 0.00 \\
550 & $20 \% \mathrm{FBH}$ & -5 & 3.00 \\
550 & $40 \% \mathrm{FBH}$ & -11 & 9.00 \\
550 & $60 \% \mathrm{FBH}$ & -9 & 20.00 \\
550 & $100 \% \mathrm{FBH}$ & 0 & 0.00 \\
\hline
\end{tabular}

Table 1. Error in the simulated voltage and phase versus experimental data

\subsection{Comparison to experimental data}

In order to compare the simulation results to experimental data, the following calibration procedure was applied: the peak magnitude and phase of the signal generated by $100 \%$ through-wall defect at each frequency was set to $20 \mathrm{~V}$ and $140^{\circ}$ respectively. This was achieved by multiplying the signal by a constant complex-valued scaling factor, which was further applied to other three signals obtained at the same frequency. Thus, the relative magnitude and phase differences between all defects were preserved. The calibrated signals for two frequencies are demonstrated on Figure 3.

Two metrics were utilized to compare the signals after calibration: the magnitude and phase of the point with the peak magnitude. The results are summarized in Table 1.
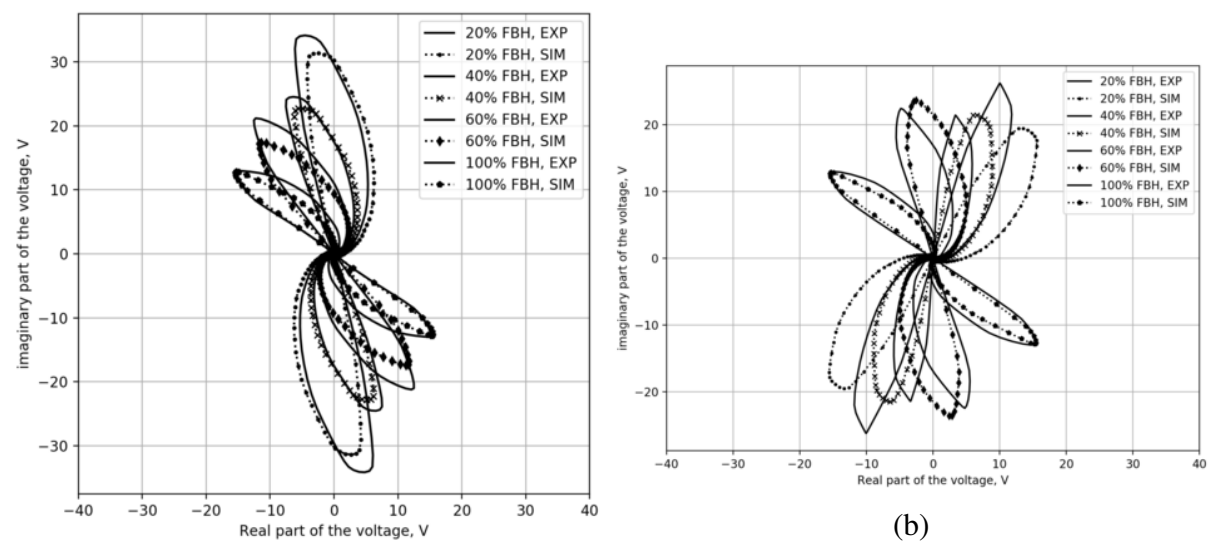

(b)

(a)

Figure 3. Calibrated differential voltage measurement, real versus imaginary part, (a) $140 \mathrm{kHz}$, (b) $280 \mathrm{kHz}$ 


\section{Conclusions}

This paper presents a numerical validation for the Reduced Vector Potential formulation with a DtN boundary condition against an experimental data. This is a differential formulation with an exact non-local boundary condition, which allows an efficient numerical implementation [8], [9].

A typical eddy current inspection problem was simulated: a differential bobbin coil scan inside a conductive tube with a volumetric defect in the wall. The simulation results show a good match with the experimental data, both in magnitude, phase and the overall shape of the signals. As could be seen in Table 1, the results are better for the lower frequency data due to the larger skin depth. Thus, generation of a finer mesh for higher frequency simulations could improve the numbers. However, the perfect match could not be achieved due to discrepancies introduced by the data acquisition system.

The presented analysis allows to conclude that the proposed formulation could be readily applied to simulate the eddy current inspection procedure.

\section{References}

[1] O. Bíró, "Edge element formulations of eddy current problems," Computer Methods in Applied Mechanics and Engineering, vol. 169, no. 3, pp. 391-405, 1999.

[2] Z. Zeng, L. Udpa, S. S. Udpa, and M. S. C. Chan, "Reduced magnetic vector potential formulation in the finite element analysis of eddy current nondestructive testing," IEEE Transactions on Magnetics, vol. 45, no. 3, pp. 964-967, 2009.

[3] A. Bossavit and J. Verite, "The "trifou" code: Solving the 3-d eddy-currents problem by using h as state variable," IEEE Transactions on Magnetics, vol. 19, no. 6, pp. 2465-2470, 1983.

[4] J. C. VERITE, "Application of a 3-d eddy current code (trifou) to non-destructive testing," COMPEL - The international journal for computation and mathematics in electrical and electronic engineering, vol. 3, no. 3, pp. 167-178, 1984.

[5] D. Givoli and J. B. Keller, "A finite element method for large domains," Computer Methods in Applied Mechanics and Engineering, vol. 76, no. 1, pp. 41-66, 1989.

[6] D. Givoli, I. Patlashenko, and J. B. Keller, "Discrete dirichlet-to-neumann maps for unbounded domains," Computer Methods in Applied Mechanics and Engineering, vol. 164, no. 1, pp. 173-185, 1998.

[7] A. Efremov, S. Ventre, L. Udpa, and A. Tamburrino, "Reduced vector potential formulation with dtn boundary condition for eddy current problems," book of abstracts, 23rd Int. Workshop on Electromagnetic Nondestructive Evaluation, 2018.

[8] A. Efremov, A. Perrotta, S. Ventre, L. Udpa, and A. Tamburrino, "Fem formulation with dirichlet-toneumann map boundary condition for eddy current problems," the 22nd International Conference on the Computation of Electromagnetic Fields, 2019.

[9] A. Efremov, A. Perrotta, S. Ventre, L. Udpa, and A. Tamburrino, "Sparsification of the dtn operator and its applications for eddy current problems," book of abstracts, 24th Int. Workshop on Electromagnetic Nondestructive Evaluation, 2019.

[10] J. Jackson, Classical Electrodynamics. John Wiley \& Sons, 1999.

[11] P. Moore and S. Udpa, Nondestructive Testing Handbook: Electromagnetic Testing. Nondestructive testing handbook, Amer Society for Nondestructive, 2004.

[12] C. Geuzaine and J.-F. Remacle, "Gmsh: A 3-D finite element mesh generator with built-in pre- and postprocessing facilities," International Journal for Numerical Methods in Engineering, vol. 79, pp. 1309 1331, 2009. 